\title{
Robotic paravertebral schwannoma resection at extreme locations of the thoracic cavity
}

\author{
Giacomo Pacchiarotti, MS, ${ }^{1}$ Michael Y. Wang, MD, ${ }^{2}$ John Paul G. Kolcun, BS, ${ }^{2}$ \\ Ken Hsuan-kan Chang, MD, ${ }^{2}$ Motasem AI Maaieh, MD, ${ }^{3}$ Victor S. Reis, MD, ${ }^{4}$ and \\ Dao M. Nguyen, MD ${ }^{4}$
}

\begin{abstract}
${ }^{1}$ Institute of Neurosurgery, University of Rome "La Sapienza," Rome, Italy; and Departments of ${ }^{2}$ Neurological Surgery and ${ }^{3}$ Orthopaedic Surgery, and ${ }^{4}$ Thoracic Surgery Section, Department of Surgery, University of Miami Miller School of Medicine, Miami, Florida
\end{abstract}

\begin{abstract}
Solitary paravertebral schwannomas in the thoracic spine and lacking an intraspinal component are uncommon. These benign nerve sheath tumors are typically treated using complete resection with an excellent outcome. Resection of these tumors is achieved by an anterior approach via open thoracotomy or minimally invasive thoracoscopy, by a posterior approach via laminectomy, or by a combination of both approaches. These tumors most commonly occur in the midthoracic region, for which surgical removal is usually straightforward. The authors of this report describe 2 cases of paravertebral schwannoma at extreme locations of the posterior mediastinum, one at the superior sulcus and the other at the inferior sulcus of the thoracic cavity, for which the usual surgical approaches for safe resection can be challenging. The tumors were completely resected with robot-assisted thoracoscopic surgery. This report suggests that single-stage anterior surgery for this type of tumor in extreme locations is safe and effective with this novel minimally invasive technique.

https://thejns.org/doi/abs/10.3171/2017.2.FOCUS16551
\end{abstract}

KEY WORDS robotic surgery; da Vinci robot; paravertebral schwannoma; posterior mediastinal neurogenic tumor

$\mathrm{T}$ YPICAL solitary posterior mediastinal neurogenic tumors (PMNTs) are paravertebral masses arising from thoracic nerve roots, sympathetic trunks, or intercostal nerves. The majority of PMNTs (95\%) are schwannomas, which are benign tumors of the nerve sheath, composed exclusively of Schwann cells., ${ }^{7,223}$ The most frequently reported location for spinal schwannomas in the current literature is the cervical region, followed by the thoracic and lumbar segments. ${ }^{8,11,18}$ Schwannomas can cause pain, paresthesias, or motor weakness, but the diagnosis is sometimes an incidental finding of an asymptomatic mass on radiological imaging such as radiography, ultrasonography, CT, or MRI. ${ }^{20}$ The prognosis of schwannoma is favorable as the tumor is usually well encapsulated and thus lends itself to complete resection, although this is not always feasible. ${ }^{3,6}$

Spinal schwannomas can be classified according to their anatomical location as intradural or extradural or as dumbbell-shaped tumors with both intradural and extradural components. Resection of spinal schwannoma is achieved by a transthoracic anterior approach via posterolateral thoracotomy, by a posterior approach via laminectomy with or without facetectomy, or by a combination of both approaches. Additional spinal instrumentation and fixation may be required if the spine is destabilized. ${ }^{1,15}$

Minimally invasive video-assisted thoracoscopic surgery (VATS) and, more recently, robot-assisted VATS (da Vinci Surgical System, Intuitive Surgical Inc.) for various intrathoracic procedures, particularly pulmonary lobectomy for lung malignancy, have been unequivocally shown to be superior to open thoracotomy with a significant reduction in postoperative morbidity, mortality, and length of stay, translating into increased cost-effectiveness with similar oncological outcomes. ${ }^{26}$ Robotic endoscopic surgery has been widely and successfully adopted by many subspecialties. By virtue of the multiple operating angles of its 4 dexterous robotic arms, 10× optical magnification, and 3D high-resolution imaging, the da Vinci Surgical System allows the surgeon to perform precise dissections from extreme angles, even for a deep target or within a 

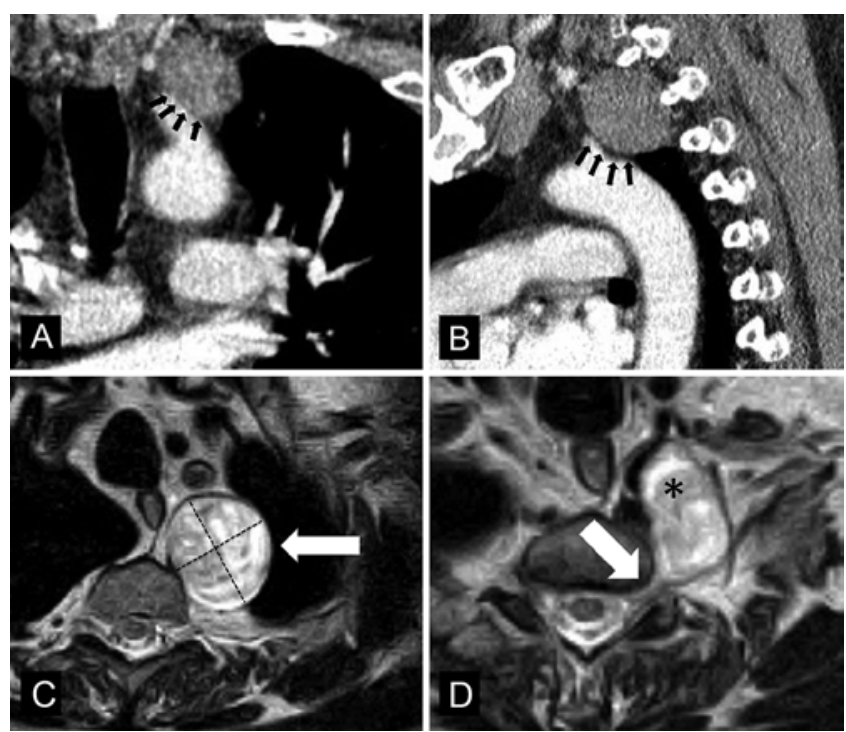

FIG. 1. Representative coronal (A) and sagittal (B) CT scans as well as axial MR images ( $C$ and $\mathbf{D}$ ) demonstrate a left paravertebral schwannoma in the superior sulcus location. The tumor closely abuts the esophagus, vertebral body, and intrathoracic subclavian artery (black and white arrows in A-C). Axial MR image shows the origin of this neurogenic tumor arising from a nerve root (white arrow, D).

narrow space. Such characteristics make this technology, as compared with VATS, extremely attractive for intrathoracic procedures such as lung lobectomy or resection of mediastinal tumors. The da Vinci robot has recently been used to treat posterior mediastinal schwannomas in a single-stage operation with excellent surgical outcomes. ${ }^{4,5,19}$ However, most existing reports on robotic schwannoma resection have involved the midthoracic region. Here, we present 2 illustrative cases of thoracoscopic schwannoma resection at the cranial and the caudal extremes of the chest cavity using the da Vinci robotic system. An accompanying video demonstrates the nuances of the technique (Video 1).

VIDEO 1. Paravertebral schwannoma resection at extremes of the thoracic cavity using the da Vinci robotic system. The video demonstrates nuances of the technique. Copyright University of Miami
Miller School of Medicine. Published with permission. Click here to view.

\section{Illustrative Cases \\ Case 1 \\ Clinical History}

This 64-year-old man was initially evaluated elsewhere following a motor vehicle accident. During the diagnostic workup, CT revealed a paraspinal left-sided thoracic mass in the superior posterior sulcus adjacent to the aortic arch and the subclavian vessel (Fig. 1). The appearance of the mass on MRI was consistent with a PMNT. It was determined that the mass would be best resected via a transthoracic anterior approach with left-sided robot-assisted thoracoscopy. Alternatively, a transaxillary thoracotomy would be needed to gain access for the resection since standard VATS would be very challenging with a high risk of conversion to open thoracotomy because of its inherent technical limitations. Informed consent was obtained to perform robotic thoracoscopic resection of the left superior sulcus schwannoma.

\section{Operative Intervention}

Standard anesthesia preparation was made for a leftsided thoracoscopy (Video 1). Lung isolation was achieved with an endobronchial balloon (Rusch EZ-Blocker, Teleflex Inc.). The patient was placed in the right lateral decubitus position, and the operative field was established as usual. The seventh intercostal space was identified, and multiple skin incisions for port access were made at predetermined sites along the space. The camera port was made first, and $\mathrm{CO}_{2}$ insufflation was used to completely deflate the left lung and gently displace the mediastinum to the contralateral side; the remaining port placements were made under direct vision. The da Vinci Xi robot was docked to the patient. The lung was retracted from the posterior superior mediastinum. The posterior thoracic compartment was visualized (Fig. 2 right). The tumor was located in the left superior sulcus, away from the aortic arch and posterior to the intrathoracic portion of the subclavian artery, abutting the sympathetic chain, the lateral aspect of the second vertebral body, and the inner arch of the first rib.
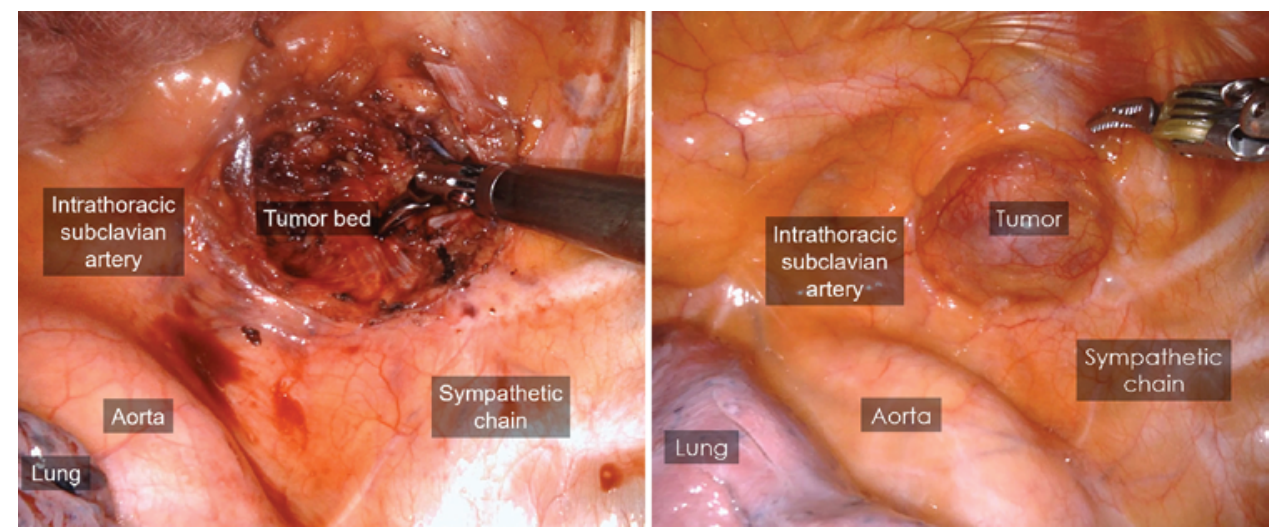

FIG. 2. Operative photographs of the left superior sulcus paravertebral schwannoma obtained before (right) and after (left) resection, showing the tumor close to the intrathoracic subclavian artery, sympathetic chain, and origins of the first and second ribs. Insufflation of $\mathrm{CO}_{2}$ displaced the mediastinum and separated the tumor from the arch of the aorta. 

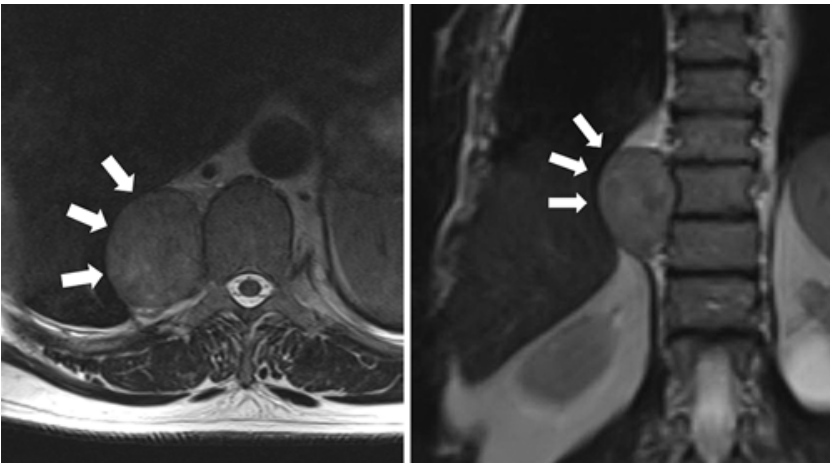

FIG. 3. Representative axial (left) and coronal (right) MR images of a right inferior sulcus paravertebral schwannoma, showing tumor abutting the T-9, T-10, and T-11 vertebral bodies as well as the diaphragm.

The mediastinal pleura lying over the tumor was divided. The mass was dissected away from surrounding structures with bipolar cautery and removed in toto with an intact capsule. There was no injury to any nearby nerve or vessel. Meticulous hemostasis was achieved and verified (Fig. 2 left). Total intraoperative blood loss was $20 \mathrm{ml}$. The robot was de-docked from the patient. A chest tube was inserted, and the patient's lung was reinflated under direct vision. The operative time was 105 minutes (15 minutes for docking of the robot and 75 minutes of robotic operating time).

\section{Postoperative Course}

The patient's postoperative course was uneventful. He had no Horner's syndrome upon discharge or at the follow-up visit on postoperative Day 12. The chest tube was removed a few hours after surgery, and the chest radiograph was normal after removal of the chest tube. The patient was discharged on postoperative Day 1 . The final pathology of this lesion was benign schwannoma, completely resected with an intact capsule.

\section{Case 2}

\section{Clinical History}

This 57-year-old man had a long (many years) history of kidney stones. Four years earlier, while undergoing investigation for kidney stones at an outside institution, he was found to have a right lower thoracic paravertebral "cyst" on abdominal CT containing images of the lower chest. Approximately 6 months ago, follow-up abdominal CT revealed enlargement of this cyst. He was referred to our institution for further evaluation. His physical examination was totally unremarkable. Chest CT demonstrated a right, lower thoracic, paravertebral $5.0 \times 4.4-\mathrm{cm}$ mass with a smooth border. It spanned 3 vertebral bodies from T-9 to T-11 with its epicenter at T-10. Thoracic MRI showed an enhancing, well-defined, $5.2 \times 4 \times 4.5-\mathrm{cm}$ paraspinal solid mass (Fig. 3). Preoperative core needle biopsy confirmed that this lesion was a schwannoma. We determined that an anterior transthoracic approach would be the most optimal for safe resection. Robot-assisted thoracoscopy instead of VATS was selected to exploit its superior 3D imaging, angulated operative arms, and intrapleural $\mathrm{CO}_{2}$ insufflation to achieve caudal displacement of the diaphragm for wide exposure of the tumor in the inferior sulcus of the thoracic cavity.

\section{Operative Report}

The standard anesthesia preparation was made for a right-sided thoracoscopy. Lung isolation was achieved with an endobronchial balloon (Rusch EZ-Blocker). The patient was placed in the left lateral decubitus position, and the operative field was established as usual. The seventh intercostal space was identified, and multiple skin incisions for port access were made at predetermined sites along this space. The camera port was made first, and $\mathrm{CO}_{2}$ insufflation was used to completely deflate the right lung and gently displace the visceral compartment of the mediastinum to the contralateral side and the diaphragm was displaced downward; the remaining port placements were made under direct vision. The da Vinci Xi robot was docked to the patient. The lung was retracted from the posterior inferior mediastinum to expose the large paraspinal mass. The tumor and adjacent organs (diaphragm, thoracic spine, aorta, and azygos vein) were visualized. The tumor was noted to be hypervascular (Fig. 4 right). The surrounding mediastinal pleura was divided to allow extrapleural dissection and detachment of the mass with its intact capsule from the underlying periosteum and segmental intercostal vein and artery. There was no injury to any nearby nerve or vessel. Meticulous hemostasis was achieved and verified (Fig. 4 left). Total blood loss was 80
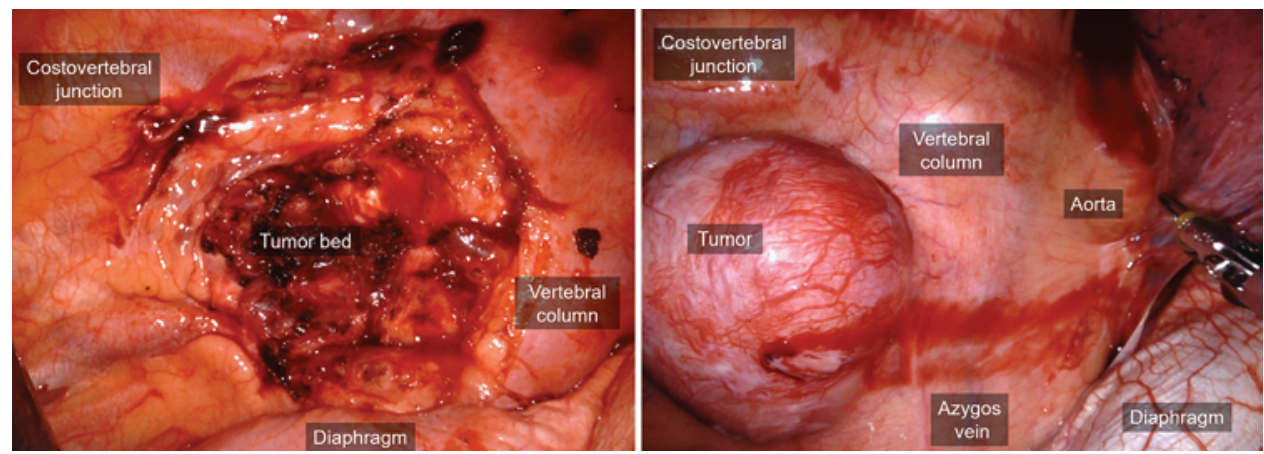

FIG. 4. Operative photographs of the right inferior sulcus paravertebral tumor obtained before (right) and after (left) resection, showing tumor covered by a hypervascular parietal pleura, adjacent to an azygos vein and descending aorta, and abutting the T-10 vertebral body. The diaphragm was displaced downward and away from the tumor with intrapleural insufflation of $\mathrm{CO}_{2}$. 
$\mathrm{ml}$. The robot was de-docked from the patient. A chest tube was inserted, and the patient's lung was reinflated under direct vision. The total operating time was $110 \mathrm{~min}$ utes (13 minutes of robot docking time and 90 minutes of robotic operating time).

\section{Postoperative Course}

The patient's postoperative course was uneventful. The chest tube was removed a few hours after completion of the operation. The patient was discharged home on postoperative Day 1. He was perfectly fine when seen on a routine outpatient visit on postoperative Day 7 . The final pathology of this lesion was benign schwannoma, completely resected with an intact capsule.

\section{Discussion}

Most spinal and paraspinal neurogenic tumors are benign nerve sheath tumors. They are predominantly schwannomas and, less frequently, neurofibromas. Most nerve sheath tumors contain both intraspinal and extraspinal portions and are often identified as spinal dumbbell tumors..$^{15}$ Several different classifications for spinal dumbbell tumors have been proposed in the literature, based on the size, location, and anatomical distribution of intraspinal (including intra- and extradural parts) and extraspinal components..$^{1,15,22}$ The surgical approach to spinal dumbbell tumors is usually tailor-made for different tumor types. Techniques include posterior laminectomy only, posterior laminectomy with facetectomy and instrumentation, and a combined posterior/anterior approach. As most dumbbell tumors consist of both intra- and extraspinal parts, thoracic surgeons and neurosurgeons commonly cooperate on spinal dumbbell tumors in thoracic segments. Only $5 \%-10 \%$ of dumbbell tumors are solely extraspinal, and these are often classified as foraminal and paravertebral. ${ }^{15}$

The foraminal and paravertebral types of spinal dumbbell tumors usually comprise a relatively large paravertebral mass in the posterior mediastinum with mild and late-onset symptoms. The 2 schwannomas in the present report best correspond to this classification since they lacked components in the spinal canal. When a foraminal and paravertebral spinal dumbbell tumor is predominantly within the posterior mediastinum, it can also be defined anatomically as a posterior mediastinal tumor. Posterior mediastinal nerve sheath tumor resection was traditionally approached anteriorly with a thoracotomy. Krätzig et al. have reported a nearly $100 \%$ success rate in completely resecting large $(>2.5 \mathrm{~cm})$ foraminal and paravertebral schwannoma using a 1-stage anterior approach. ${ }^{10}$ Posterior mediastinal nerve sheath tumors are very amenable to standard transthoracic approaches via either open thoracotomy or minimally invasive thoracoscopy. The most challenging locations for safe resection are the extreme superior and inferior sulci of the thoracic cavity by virtue of the confined anatomical space severely limiting the ability to visualize and to identify important adjacent structures (e.g., subclavian vessels, inferior border of the brachial plexus of the superior sulcus, azygos vein, esophagus, descending aorta, thoracic duct, segmental intercostal vessels, and diaphragm of the deep inferior sulcus).
Our group has extensive experience with thoracic robotic surgery since adopting this innovative technology more than 4 years ago. We have performed 500 intrathoracic procedures, $15 \%$ of which involved resection of mediastinal tumors, and have been using the third-generation da Vinci Xi system since 2014. Many features of robot-assisted thoracoscopic surgery facilitate safe complete resection of PMNTs, particularly those in extreme locations of the thoracic sulcus such as those described in this report. The use of $\mathrm{CO}_{2}$ insufflation and the 3D high-definition imaging of the robotic system enable complete visualization of the tumors as well as important adjacent anatomical structures. Seven-degree movement of the tip of the robotic arms, interchangeable camera ports, extreme movements of the robotic arms, and bipolar electrocautery allow precise dissection of tumors from important structures, even in very confined anatomical spaces. We have standardized our port placement and robotic docking techniques, and given our experience, nothing had to be done differently in the 2 illustrative cases to accommodate the difficult tumor locations. We had no technical difficulty performing the resections, and the robotic operating time was very acceptable at $<100$ minutes. The robotic docking time of 12-15 minutes is our current standard. The minimally invasive thoracic surgery platform allows rapid postoperative recovery, with pain readily controlled by oral opioid analgesics, and early discharge from the hospital without compromising the oncological principle of complete resection of tumors with an intact capsule. Discharge home on postoperative Day 1 is feasible in more than $80 \%$ of our patients undergoing robot-assisted thoracoscopic resection of mediastinal tumors.

Robot-assisted thoracoscopic resection of posterior mediastinal schwannomas has been previously reported, generally at midthoracic levels or in the inferior mediastinum. ${ }^{4,5,19}$ To our knowledge, there has been no description in the literature regarding this approach applied to neurogenic tumors at the extreme locations (the very top and bottom of the posterior mediastinum). Indeed, the presence of schwannomas in these regions is rare. One recent report did suggest a similar presentation and approach; however, the description of the tumor location and pathology was unclear. ${ }^{25}$ Robot-assisted thoracoscopy has also been applied in combined procedures in which tumor location or morphology has required both anterior and posterior resection. ${ }^{16}$ While robotics has found growing favor in a variety of surgical disciplines, it has yet to find broad utilization in spinal neurosurgery. The few applications to date have been largely restricted to spinal instrumentation. 2, $, 17,27$ The clinical, institutional, and economic benefits of minimally invasive surgery have been extensively described in the neurosurgical literature. ${ }^{13,14,21,24}$ The 2 cases featured in this report demonstrate that robot-assisted thoracoscopic resection is an effective therapy for certain paravertebral schwannomas in both the high superior and low inferior posterior mediastinum with excellent perioperative outcomes.

\section{References}

1. Asazuma T, Toyama Y, Maruiwa H, Fujimura Y, Hirabayashi $\mathrm{K}$ : Surgical strategy for cervical dumbbell tumors based on 
a three-dimensional classification. Spine (Phila Pa 1976) 29:E10-E14, 2004

2. Beutler WJ, Peppelman WC Jr, DiMarco LA: The da Vinci robotic surgical assisted anterior lumbar interbody fusion: technical development and case report. Spine (Phila Pa 1976) 38:356-363, 2013

3. Biswas D, Marnane CN, Mal R, Baldwin D: Extracranial head and neck schwannomas-a 10-year review. Auris Nasus Larynx 34:353-359, 2007

4. Cerfolio RJ, Bryant AS, Minnich DJ: Operative techniques in robotic thoracic surgery for inferior or posterior mediastinal pathology. J Thorac Cardiovasc Surg 143:1138-1143, 2012

5. Chon SH, Shinn SH, Song DS, Jeong MA: Double primary tumor, thymic mass and posterior mediastinal neurogenic tumor, in a patient with acute pancreatitis performed with single-staged robotic-assisted thoracoscopic surgery. Surg Laparosc Endosc Percutan Tech 20:e176-e178, 2010

6. Conti P, Pansini G, Mouchaty H, Capuano C, Conti R: Spinal neurinomas: retrospective analysis and long-term outcome of 179 consecutively operated cases and review of the literature. Surg Neurol 61:34-44, 2004

7. Fierro N, Morelli A, Del Grammastro A, D’Ermo G, Di Cola G, Annessi M, et al: [Posterior mediastinum neoplasms: a case of schwannoma.] G Chir 25:35-38, 2004 (Ital)

8. Jinnai T, Koyama T: Clinical characteristics of spinal nerve sheath tumors: analysis of 149 cases. Neurosurgery 56:510 515,2005

9. Kim MJ, Ha Y, Yang MS, Yoon DH, Kim KN, Kim H, et al: Robot-assisted anterior lumbar interbody fusion (ALIF) using retroperitoneal approach. Acta Neurochir (Wien) 152:675-679, 2010

10. Krätzig T, Dreimann M, Klingenhöfer M, Floeth FW, Krajewski K, Eicker SO: Treatment of large thoracic and lumbar paraspinal schwannoma. Acta Neurochir (Wien) 157:531538,2015

11. Kyoshima K, Horiuchi T, Zenisaka H, Nakazato F: Thoracic dumbbell intra- and extramedullary schwannoma. J Clin Neurosci 12:481-484, 2005

12. Marchevsky AM: Mediastinal tumors of peripheral nervous system origin. Semin Diagn Pathol 16:65-78, 1999

13. McGirt MJ, Parker SL, Lerner J, Engelhart L, Knight T, Wang MY: Comparative analysis of perioperative surgical site infection after minimally invasive versus open posterior/ transforaminal lumbar interbody fusion: analysis of hospital billing and discharge data from 5170 patients. J Neurosurg Spine 14:771-778, 2011

14. Ohya J, Oshima Y, Chikuda H, Oichi T, Matsui H, Fushimi $\mathrm{K}$, et al: Does the microendoscopic technique reduce mortality and major complications in patients undergoing lumbar discectomy? A propensity score-matched analysis using a nationwide administrative database. Neurosurg Focus 40(2):E5, 2016

15. Ozawa H, Kokubun S, Aizawa T, Hoshikawa T, Kawahara C: Spinal dumbbell tumors: an analysis of a series of 118 cases. J Neurosurg Spine 7:587-593, 2007

16. Perez-Cruet MJ, Welsh RJ, Hussain NS, Begun EM, Lin J, Park P: Use of the da Vinci minimally invasive robotic system for resection of a complicated paraspinal schwannoma with thoracic extension: case report. Neurosurgery 71 (1 Suppl Operative):209-214, 2012

17. Ponnusamy K, Chewning S, Mohr C: Robotic approaches to the posterior spine. Spine (Phila Pa 1976) 34:2104-2109, 2009

18. Riffaud L, Morandi X, Massengo S, Carsin-Nicol B, Heresbach N, Guegan Y: MRI of intramedullary spinal schwanno- mas: case report and review of the literature. Neuroradiology 42:275-279, 2000

19. Ruurda JP, Hanlo PW, Hennipman A, Broeders IA: Robotassisted thoracoscopic resection of a benign mediastinal neurogenic tumor: technical note. Neurosurgery 52:462-464, 2003

20. Saint-Blancard P, Harket A, Bonnichon A, Jancovici R: [Neurogenic spindle-cell tumors of the mediastinum: two cases.] Presse Med 37:229-234, 2008 (Fr)

21. Shamji MF, Goldstein CL, Wang M, Uribe JS, Fehlings MG: Minimally invasive spinal surgery in the elderly: does it make sense? Neurosurgery 77 (Suppl 4):S108-S115, 2015

22. Sridhar K, Ramamurthi R, Vasudevan MC, Ramamurthi B: Giant invasive spinal schwannomas: definition and surgical management. J Neurosurg 94 (2 Suppl):210-215, 2001

23. Strollo DC, Rosado-de-Christenson ML, Jett JR: Primary mediastinal tumors: part II. Tumors of the middle and posterior mediastinum. Chest 112:1344-1357, 1997

24. Vertuani S, Nilsson J, Borgman B, Buseghin G, Leonard C, Assietti R, et al: A cost-effectiveness analysis of minimally invasive versus open surgery techniques for lumbar spinal fusion in Italy and the United Kingdom. Value Health 18:810-816, 2015

25. Xu S, Liu B, Wang X, Meng H, Wang T, Xu W, et al: Robotic thoracic surgery of the posterior superior mediastinal mass. Ann Transl Med 3:127, 2015

26. Yang CF, Sun Z, Speicher PJ, Saud SM, Gulack BC, Hartwig $\mathrm{MG}$, et al: Use and outcomes of minimally invasive lobectomy for Stage I non-small cell lung cancer in the National Cancer Data Base. Ann Thorac Surg 101:1037-1042, 2016

27. Yang MS, Yoon DH, Kim KN, Kim H, Yang JW, Yi S, et al: Robot-assisted anterior lumbar interbody fusion in a Swine model in vivo test of the da Vinci surgical-assisted spinal surgery system. Spine (Phila Pa 1976) 36:E139-E143, 2011

\section{Disclosures}

Dr. Wang is a consultant for and holds a patent with DePuy Spine, is a consultant for K2M and Aesculap, and has direct stock ownership in ISD.

\section{Author Contributions}

Conception and design: Chang, Wang. Acquisition of data: Chang, Pacchiarotti, Kolcun, Reis. Analysis and interpretation of data: Chang, Pacchiarotti, Wang, Kolcun. Drafting the article: Chang, Pacchiarotti, Wang, Kolcun, Reis. Critically revising the article: Chang, Wang, Kolcun, Nguyen. Reviewed submitted version of manuscript: Chang, Wang. Approved the final version of the manuscript on behalf of all authors: Chang. Administrative/ technical/material support: Chang, Pacchiarotti, Wang, Al Maaieh, Reis, Nguyen. Study supervision: Chang, Wang, Al Maaieh, Nguyen.

\section{Supplemental Information \\ Videos}

Video 1. https://vimeo.com/207448024.

\section{Correspondence}

Ken Hsuan-kan Chang, Department of Neurological Surgery, Lois Pope LIFE Center, 1095 NW 14th Terrace, Miami, FL 33136. email: hsuankanchang@gmail.com. 